\title{
Reverse Takotsubo Cardiomyopathy from Myasthenic Crisis: A Case Report
}

\author{
Anub John ${ }^{1 *}$, Sanjeev Singh ${ }^{1}$, Angelica Singh ${ }^{2}$ and Carrie G Lenneman ${ }^{1,3,4}$ \\ ${ }^{1}$ Department of Internal Medicine, University of Louisville, Kentucky, USA \\ ${ }^{2}$ Stony Brook University, New York City, New York, USA \\ ${ }^{3}$ Division of Cardiology, University of Louisville, USA \\ ${ }^{4}$ Robley Rex Louisville VAMC, Kentucky, USA
}

*Correspondence author: Anub John, Department of Internal Medicine, University of Louisville, Kentucky, USA, Tel.: 502-777-3628; Fax: (502) 852-8980; E-mail: agjohn07@louisville.edu

Received date: July 18, 2014; Accepted date: Aug 28, 2014; Published date: Sep 04, 2014

Copyright: ( 2014 John A, et al. This is an open-access article distributed under the terms of the Creative Commons Attribution License, which permits unrestricted use, distribution, and reproduction in any medium, provided the original author and source are credited.

\begin{abstract}
A 34 year old female with undiagnosed myasthenia gravis (MG) presented with shortness of breath and weakness. She was diagnosed with community acquired pneumonia and myasthenic crisis (MC). Her hospital course was complicated by hypoxemic respiratory failure requiring intubation with echocardiography revealing a hyper-dynamic apex and basilar hypokinesis consistent with Reverse Takotsubo Cardiomyopathy (RTCM). We present the first published case of RTCM from MC and review the existing cases of stress induced cardiomyopathies in MG patients.
\end{abstract}

Keywords: Reverse Takotsubo Cardiomyopathy; Takotsubo Cardiomyopathy; Cardiomyopathy; Myasthenia gravis; Myasthenic crisis; Stress induced cardiomyopathy

\section{Abbreviations: \\ TCM: Takotsubo Cardiomyopathy; RTCM: Reverse Takotsubo Cardiomyopathy; MG: Myasthenia Gravis; MC: Myasthenic Crisis}

\section{Introduction}

Stress induced cardiomyopathy, or Takotsubo cardiomyopathy (TCM), is defined as transient left ventricular dysfunction occurring from physical or emotional stress in the absence of coronary artery disease $[1,2]$. TCM has four recognized variants including classical, reverse, mid-ventricular, and localized type [1]. Reverse Takotsubo cardiomyopathy (RTCM) is characterized by basal segment hypokinesis with hyper-dynamic apex [3]. Although myasthenia gravis (MG) is associated with classical TCM, this is the first case, to our knowledge, of RTCM in a patient with myasthenia gravis.

\section{Case}

A 34 year old Caucasian lady with a history of asthma, tobacco use and Crohn's disease presented to our emergency room from an outside hospital with shortness of breath. She reported progressively worsening shortness of breath over a month time period. Over this time span, she had multiple visits to the emergency room and was initially diagnosed with gastro-esophageal reflux disease and asthma exacerbation. She reported a productive cough with yellowish sputum and wheezing for several days that did not improve on outpatient antibiotics or steroids. During this time, she denied chest pain, left arm pain, jaw pain, diaphoresis, or any acute coronary syndrome equivalents.

The patient's acute symptoms were associated with an overall decline of health for a one year period. For the last year, the patient reported progressively worsening weakness, fatigue and dysphagia. She described the weakness as generalized and worsening at the end of the day. She had difficulty combing her hair, climbing stairs, and holding objects for a prolonged period of time with bilateral drooping eyelids. She reported dysphagia mostly to solids and then liquids, as well as an accompanying 40 pound weight loss over the past six months.

Upon presentation, the patient was placed on bi-level positive airway pressure (BiPAP) device for respiratory support. Examination revealed a young, cachectic female in respiratory distress. Vital signs on admission included a blood pressure of $122 / 78 \mathrm{~mm} \mathrm{Hg}$, pulse 74 beats per minute, respiratory rate of 25 breaths per minute and oxygen saturation of $97 \%$ while on $\mathrm{BiPAP}$ at $10 / 5$ on $40 \% \mathrm{FiO}_{2}$. She was able to follows commands and was oriented to time, place and person. Pertinent positive examination findings included a cardiovascular exam that revealed regular rhythm, S3 gallop, no murmurs, no peripheral edema, warm extremities, and no jugular venous distension. Pulmonary exam was significant for left basilar crackles. Neurological exam revealed drooping of eye lids, weakness of neck extension and weakness of muscle groups in bilateral proximal upper and lower extremities (strength 4/5), 2+ deep tendon reflexes and negative Babinski reflex. Meningeal and cerebellar signs were negative on examination. Laboratory findings included a white blood cell count of $13,000 / \mathrm{mm}^{3}$ with normal complete blood count and complete metabolic profile including renal and liver function (Table 1). D-dimer level was normal. Troponin I level was elevated at $1.4 \mathrm{ng} / \mathrm{dl}$. Creatinine kinase $(\mathrm{CK})$ was normal but she had elevated CK-MB $(6.5 \mathrm{ng} / \mathrm{ml})$ and CK-MB index (8.4). EKG done on admission showed sinus rhythm and borderline $\mathrm{T}$-wave abnormalities in the antero-lateral leads. Initial chest radiography demonstrated mild hyperinflation with left basilar infiltrates. Urine streptococcal antigen was positive. Differential diagnosis for the weakness was initially broad but included myasthenic crisis versus motor neuron disease versus muscle disease versus immunologic, infectious, toxic or CNS pathology. A bedside tensilon test was conducted and was positive. A subsequent MRI of the brain was normal and a chest CT did not reveal a thymoma. Infectious 
Page 2 of 3

workup was negative for HIV antibodies. Immunologic workup came back positive for ANA and Anti-dsDNA antibodies.

\begin{tabular}{|l|l|}
\hline Hemoglobin (gm/dl) & $12.7(11.2-15.7)$ \\
\hline White Cell Count (Thousand/mm3) & $13(4.1-10.8)$ \\
\hline Platelet count (Thousand/mm3) & $196(140-370)$ \\
\hline Serum electrolytes & Within Normal Limits \\
\hline Serum Creatinine (mg/dl) & $0.5(0.5-1.2)$ \\
\hline Liver Function Tests & Within Normal Limits \\
\hline Serum Creatine Kinase (IU/L) & $94(30-135)$ \\
\hline Serum Creatine Kinase - MB Isoenzyme (ng/ml) & $6.5(0-3.2)$ \\
\hline Troponin I (ng/ml) & $1.4(0.000-0.034)$ \\
\hline Urine Streptococcal Antigen & Positive \\
\hline Sputum Culture & Streptococcus pneumoniae \\
\hline ANA (Antinuclear antibody) & Positive \\
\hline Anti-dsDNA antibody (IU/ml) & $7(<5)$ \\
\hline AChR-Ab receptor binding antibody (nmol/L) & $33.09(<0.4)$ \\
\hline AChR-Ab receptor blocking antibody (\% inhibition) & $51(<15)$ \\
\hline HIV 1-2 antibodies & Negative \\
\hline
\end{tabular}

Table 1: Laboratory testing on admission

Given her elevated cardiac biomarkers and EKG changes, a 2-D echocardiography was done on the day of admission that showed mild to moderately reduced left ventricular systolic function and calculated left ventricular ejection fraction of $43 \%$ (Biplane Simpson's method). There was basal wall motion akinesis and apical hyperkinesis suggestive of an acute, reverse Takotsubo cardiomyopathy (Figure 1). Left ventricular diastolic function was normal. Troponin I level peaked upto $1.6 \mathrm{ng} / \mathrm{ml}$ without any further EKG changes. The patient was admitted to the intensive care unit given her respiratory distress and positive tensilon test for likely myasthenic crisis (MC). Her respiratory distress worsened and a subsequent arterial blood gas demonstrated an acute respiratory acidosis and hypoxemia. The patient was emergently intubated. She was started on pyridostigmine and underwent plasmapheresis over the next 5 days with improvement in symptoms. Her streptococcal pneumonia was treated with appropriate antibiotics. She was successfully extubated on hospital day 3. Follow-up echocardiogram on hospital day 5 demonstrated normal left ventricular ejection fraction and improving basilar wall motion. She was started on aspirin, atorvastatin and metoprolol that were stopped prior to discharge. Given her precipitating condition, absence of cardiac risk factors and improvement in echocardiographic features, an invasive ischemic workup was not pursued.

Serological tests for myasthenia gravis resulting in positive AChR$\mathrm{Ab}$ receptor binding and blocking antibodies. The patient was discharged with pyridostigmine for symptomatic therapy with outpatient neurology follow-up.

\section{Discussion}

This is the first case report, to the authors' knowledge, of MG and RTCM. An existing PUBMED review of the literature by the authors found only 8 cases of TCM and MG with no incidence of RTCM and MG until now (Table 2) [4-11]. The average age of stress induced cardiomyopathy (TCM and RTCM) was 68 with the majority of patients being female (female to male ratio of nine to one). Most stress induced cardiomyopathy occurred from MC (4/9), followed by plasmapheresis (2/9), IVIG treatment (2/9), and lastly bereavement (1/9). Our patient had undiagnosed MG. She suffered a pneumonia which caused a MC. The MC and pneumonia were the probable triggers for the RTCM.

\begin{tabular}{|c|c|c|c|c|}
\hline & Age & Sex & Trigger & Event \\
\hline Beydoun [6] & 60 & $\mathrm{~F}$ & Bereavement & TCM \\
\hline Anand [4] & 50 & $\mathrm{~F}$ & IVIG & TCM \\
\hline Gautier [7] & 82 & $\mathrm{~F}$ & IVIG & TCM \\
\hline Arai [5] & 83 & $\mathrm{~F}$ & Plasmapheresis & TCM \\
\hline $\begin{array}{l}\text { Mayor-Gomez } \\
{[9]}\end{array}$ & 83 & $\mathrm{~F}$ & Myasthenic Crisis & TCM \\
\hline Wong [11] & 64 & $\mathrm{~F}$ & Myasthenic Crisis & TCM \\
\hline Sousa [10] & 64 & M & Plasmapheresis & TCM \\
\hline Hirose [8] & 63 & $\mathrm{~F}$ & $\begin{array}{l}\text { Disopyramide precipitating } \\
\text { Myasthenic Crisis }\end{array}$ & TCM \\
\hline Our patient & 34 & $\mathrm{~F}$ & Myasthenic Crisis/Pneumonia & RTCM \\
\hline
\end{tabular}

Table 2: Takotsubo and Reverse Takotsubo Cardiomyopathy in Myasthenia Gravis Patients. TCM - Takotsubo cardiomyopathy, RTCM - Reverse Takotsubo cardiomyopathy; IVIG - Intravenous Immunoglobulin; F- Female; M-Male

Traditionally, TCM is defined as reversible or transient systolic dysfunction of the left ventricle, in the absence of obstructive coronary artery disease. The classical apical variant of TCM is more common (70 to $80 \%$ ) with RTCM accounting for $1-2 \%$ of cases [2]. Patients present with symptoms similar to acute coronary syndrome, usually secondary to severe emotional or physical stress, acute medical illness or neurological event. The pathophysiology is conjectured to involve excess catecholaminergic release causing direct myocardial toxicity, but may also involve transient coronary artery vasospasm and microvascular dysfunction [2]. More than one coronary artery territory is involved and is related to the variable distribution and density of beta- 1 and beta- 2 receptors in the myocardium. There is research attempting to differentiate TCM from acute myocardial infarction by serum levels of miRNA [12].

Although TCM is a reversible condition, mortality remains high. The acute phase has mortality rates of $4.5 \%$ with men three times more likely to die [13]. After the acute phase, mortality rates increase up to $38 \%$ with fatalities resulting from pulmonary edema, cardiogenic shock, mitral regurgitation, left ventricular outflow tract obstruction and arrhythmias [13]. Recurrence of TCM is also likely, with a recent meta-analysis stating that at least $1.5 \%$ of patients with TCM will have recurrence of the disease and that those patients with severe TCM are more likely to have recurrence. The same study demonstrated ACE inhibitors to reduce recurrence [14]. 
Citation: John A, Singh S, Singh A, Lenneman CG (2014) Reverse Takotsubo Cardiomyopathy from Myasthenic Crisis: A Case Report. J Cardiovasc Dis Diagn 2: 174. doi:10.4172/2329-9517.1000174

Page 3 of 3

\section{RTCM vs TCM}

Alongside the echocardiographic differences, there are many demographic differences between TCM and RTCM. In an analysis of 103 patients with stress induced cardiomyopathy by Song, RTCM occurred in younger patients (median age 54.5 vs 64.0) with a lower prevalence of dyspnea, cardiogenic shock, and mitral regurgitation than its TCM counterpart [15]. In a similar analysis of 60 patients by Ramaraj, the mean age of RTCM was 36 years old vs 62 years old for all other variants [1]. The younger age of RTCM is related to its pathophysiology. Since stress induced cardiomyopathy requires an adrenergic surge and younger patients have higher concentration of adrenergic receptors at their ventricular base, younger patients present with basilar hypokinesis after an acute stressor [1,15]. Indeed, our patient was much younger than her TCM counterparts reported in Figure 1, and that may largely explain her presentation as RTCM rather than TCM. As mentioned previously, TCM occurs more frequently than RTCM. This was similar to our review of MG patients as only $10 \%$ of patients experienced RTCM while $90 \%$ of patients experienced TCM. Age may have played a confounding role.
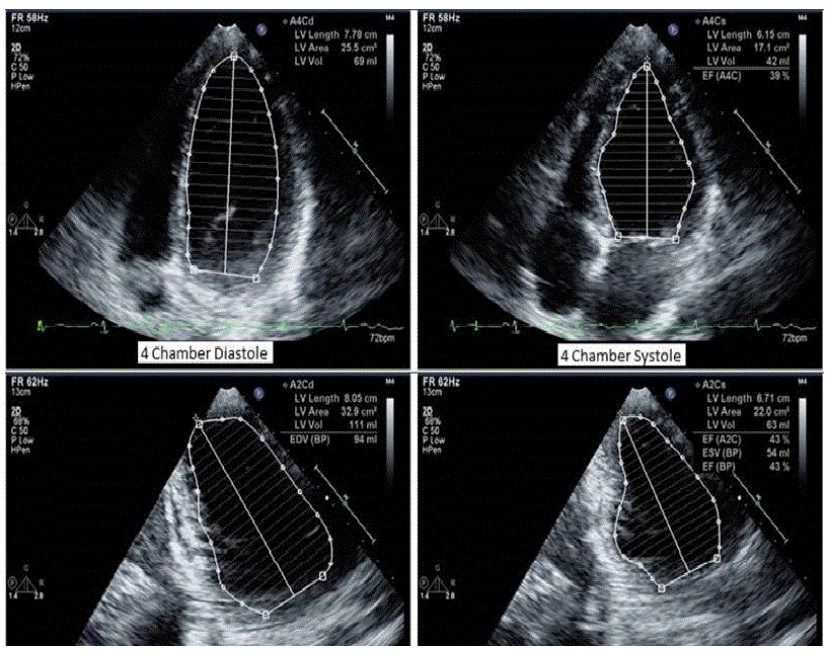

Figure 1: Four chamber and two chamber view of reverse takotsubo cardiomyopathy in diastole and systole

\section{Conclusion}

We present the first reported case of Reverse Takotsubo Cardiomyopathy in a patient with myasthenia gravis. Our patient's RTCM was precipitated by a myasthenic crisis. Her presentation as RTCM over TCM likely involved her younger age. More studies are needed to standardize the treatment and outcomes of patient with TCM and RTCM.

\section{References}

1. Ramaraj R, Movahed MR (2010) Reverse or inverted takotsubo cardiomyopathy (reverse left ventricular apical ballooning syndrome) presents at a younger age compared with the mid or apical variant and is always associated with triggering stress. Congest heart fail 16: 284-286.

2. Andrade AA, Stainback RF (2014) Takotsubo cardiomyopathy. Tex Heart Inst J 41: 299-303.

3. Haghi D, Papavassiliu T, Flüchter S, Kaden JJ, Pörner T, et al. (2006) Variant form of the acute apical ballooning syndrome (takotsubo cardiomyopathy): observations on a novel entity. Heart 92: 392-394.

4. Anand US, Viswanathan S, Arulneyam J3 (2013) Pulmonary edema in myasthenic crisis. Case Rep Crit Care 2013: 863620.

5. Arai M, Ukigai H, Miyata $H$ (2004) [A case of transient left ventricular ballooning ("Takotsubo"-shaped cardiomyopathy) developed during plasmapheresis for treatment of myasthenic crisis]. Rinsho shinkeigaku 44: 207-210.

6. Beydoun SR, Wang J, Levine RL, Farvid A (2010) Emotional stress as a trigger of myasthenic crisis and concomitant takotsubo cardiomyopathy: a case report. J Med Case Rep 4: 393.

7. Gautier P, Ravan R, Najjar M, Belhakem A, Ferrier N, et al. (2011) [TakoTsubo syndrome during normal human immunoglobolin perfusion]. Ann Cardiol Angeiol (Paris) 60: 290-295.

8. Hirose K, Yamaguchi H, Oshima Y, Choraku M, Hirono A, et al. (2008) Severe respiratory failure and torsades de pointes induced by disopyramide in a patient with myasthenia gravis. Intern Med 47: 1703-1708.

9. Mayor-Gomez S, Lacruz F, Ezpeleta D (2012) [Myasthenic crisis and Takotsubo syndrome: a non-chance relationship]. Rev Neurol 55: 725-728.

10. Sousa JM, Knobel M, Buchelle G, Sousa JA, Fisher CH, et al. (2005) [Transient ventricular dysfunction (Takotsubo cardiomyopathy)]. Arq Bras Cardiol 84: 340-342.

11. Wong CP, Chia PL (2012) Recurrent takotsubo cardiomyopathy precipitated by myasthenic crisis. Int J Cardiol 155: e11-12.

12. Jaguszewski M, Osipova J, Ghadri JR, Napp LC, Widera C, et al. (2014) A signature of circulating microRNAs differentiates takotsubo cardiomyopathy from acute myocardial infarction. Euro Heart J 35: 999-1006.

13. Singh K, Carson K, Shah R, Sawhney G, Singh B, et al. (2014) Metaanalysis of clinical correlates of acute mortality in takotsubo cardiomyopathy. Am J Cardiol 113: 1420-1428.

14. Singh K, Carson K, Usmani Z, Sawhney G, Shah R, et al. (2014) Systematic review and meta-analysis of incidence and correlates of recurrence of takotsubo cardiomyopathy. Int J Cardiol 174: 696-701.

15. Song BG, Chun WJ, Park YH, Kang GH, Oh J, et al. (2011) The clinical characteristics, laboratory parameters, electrocardiographic, and echocardiographic findings of reverse or inverted takotsubo cardiomyopathy: comparison with mid or apical variant. Clinical cardiology 34: 693-9. 